\title{
The Influence of Leader-Member Exchange on Voice Behavior: The Mediating Role of Psychological Empowerment
}

\author{
Chen Hong-an, Chu Zhi-Qiang \\ School of Business, East China University of Science and Technology, Shanghai, China \\ Email address: \\ chenha19@163.com (Chen Hong-an), zhiqiang21104@163.com (Chu Zhi-Qiang)
}

To cite this article:

Chen Hong-an, Chu Zhi-Qiang. The Influence of Leader-Member Exchange on Voice Behavior: The Mediating Role of Psychological Empowerment. Science Innovation. Vol. 4, No. 4, 2016, pp. 199-204. doi: 10.11648/j.si.20160404.15

Received: August 24, 2016; Accepted: September 19, 2016; Published: September 19, 2016

\begin{abstract}
Voice behavior refers to that employee to express their views, opinions and information related to their work. Many researches show that voice behavior has significant importance on the prevention of decision-making mistakes and the improvement of organizational effectiveness. Based on the survey of 216 employees, the paper explores the influence and mechanisms of LMX and two forms of voice behavior. The results show that LMX has significant positive impacts on both promotive and prohibitive voice behavior. And psychological empowerment partially mediates the relation between LMX and two forms of voice behaviors.
\end{abstract}

Keywords: LMX, Psychological Empowerment, Promotive Voice, Prohibitive Voice

\section{领导一成员交换对员工建言的影响: 心理授权的中介作用}

\section{陈洪安, 褚志强}

商学院, 华东理工大学, 上海市, 中国

\section{邮箱}

chenha19@163. com(陈洪安), zhiqiang21104@163.com(褚志强)

摘要：建言行为是指员工表达其对工作相关的观点、意见及信息。诸多研究表明员工建言行为对预防决策失误、提升 组织效能等方面具有重要意义。基于216名员工的调查, 本文检验了领导一成员交换（leader-member exchange，LMX） 对促进性建言和抑制性建言的影响及作用机制。结果表明, LMX对员工促进性建言、抑制性建言有显著的正向影响; 心理授权在LMX与促进性建言、抑制性建言之间具有部分中介作用。

关键词：领导一成员交换, 心理授权, 促进性建言, 抑制性建言

\section{1. 引言}

随着经济全球化的不断发展, 企业所面对的外部环境 复杂性与不确定性日益增强, 企业面临的市场竞争也愈发 激烈。仅仅依赖于管理层的智慧已不足以解决企业生存与 发展中的所有问题。因而充分发挥员工智慧, 让他们为企
业发展献言献策的重要性不言而喻 $[1]$ 。实际上, 很多学 者已将员工主动性视为组织竞争优势乃至成功的来源 [2]。 建言行为是一种典型的主动性行为已经为国内外学者广 泛关注。然而现实的状况是, 组织中员工对建言行为表现 谨慎, 甚至会出现 “知而不言” 的局面 [3]。这种现状在 中国情景中表现的更为普遍。如何激发员工建言行为, 从 
而为组织发展献言献策, 已经成为中国企业函需解决的重 要问题。

实际上, 领导作为员工建言的主要对象, 在员工建言 的过程中扮演着重要角色。而现有关于员工建言前因变量 的研究大多聚焦于个体特征和情境因素, 前者关注员工个 体特征的差异后者则强调不同的领导风格 [4]。鲜有研究 从领导与员工互动的视角探究领导一成员交换对员工建 言行为的影响机制。建言行为是由员工主动发起, 是一种 发生领导与员工之间的互动行为。领导与员工之间的关系 决定着互动的效果, 可以合理预计LMX是影响员工建言行 为的重要因素。

此外, 在LMX影响员工建言行为的过程中, 心理授权 无疑是重要的中介因素。心理授权是个体体验到的被授权 的心理状态与认知综合体, 已被证实会对员工行为产生重 要影响。凌俐等指出, 心理授权是揭示变革型领导、LMX 等变量内在作用机制的重要因素, 是这些变量对工作态度、 工作行为等相关结果变量发挥作用的重要中介变量 [5]。

综上所述, 本文将探讨LMX对员工建言行为的影响, 以及心理授权在LMX和员工建言行为间的中介效应, 旨在 为激发中国企业员工建言提供有益理论依据和现实指导。

\section{2. 文献回顾与研究假设}

\section{1. LMX与建言行为}

领导成员交换理论是在互惠规范理论以及社会交换 理论等基础上发展而来, Graen等(1972) 认为由于领导者 的时间、资源限制, 领导者会以不同的方式区别对待下属, 因而会产生 “圈内成员” 与 “圈外成员” 的差别 [6]。对 于圈内成员, 他们与领导者保持着高质量的交换关系, 被 领导视为自己人，他们得到领导更多的支持、关注及良好 的工作评价等, 作为交换, 这些圈内成员对领导报以信任、 尊重和喜欢。对于圈外员工, 他们与领导在正式的权力与 规则下保持正式的工作关系，他们与领导交换关系缺乏积 极互动, 具有较高的任务导向性。相关的实证研究证实在 组织环境中, 圈内圈外员工确实存在 [7]。

有关员工建言行为的研究起始于1970年的Hirschman, 他认为当员工对工作不满意时可能会用建言代替离职。这 是研究建言行为中具有代表性的流派之一, 该流派将建言 行为视为员工对工作感到不满意以及组织中存在问题时 的建设性反应。另一流派将建言行为视为角色外行为或组 织公民行为。例如, Van Dyne和LePine (1998) 认为建言是 一种角色外行为, 是员工表达自己对组织中问题和观点与 建议 [8]。Van Dyne (2003) 等认为建言行为以合作为动机 表达和工作相关的信息、观点及意见, 是组织公民行为的 一种 [9]。关于建言行为研究的这两大派别中, 后者对建 言行为的定义得到学者的普遍认同。在此基础上学者们, 对建言行为定义和维度划分进一步研究。其中具有代表性 的如, Liang等 (2008) 提出了一个二维建言模型, 即促 进性建言和抑制性建言, 其中促进性建言是指员工为提高 组织效率主动提出意见建议。抑制性建言则是指员工针对 组织运行中存在的问题提出看法 [10]。
领导是员工建言的主要对象, LMX是影响员工建言行 为的关键因素之一。如前所述, 领导者会对以差异化的方 式与员工建立交换关系。对于那些与领导建立高质量交换 关系的圈内员工，他们获得上级更多的信任、支持以及更 好的绩效评价和晋升机会。作为交换, 这些圈内员工也更 有动机承担更多的工作职责, 甚至包括部分职责之外的工 作。同时，圈内员工被领导视为 “自己人”，他们拥有更 多机会与领导沟通、交流的机会。这些都无疑会激发圈内 员工的建言行为。

相关实证研究也支持这一推理, Gerster和Day (1997) 的元分析表明领导一成员交换与员工角色外行为之间存 在着显著的正相关关系 [11]。国内学者汪林、储小平等 (2008) 以中国家族企业为研究对象, 证实领导部属交换 对员工组织公民行为有正向的预测效果 [12]。进一步, 钱 士茹等 (2015) 也证实领导部属交换通过内部人身份认知 对员工建言有正向影响 [13]。舟霞等 (2015) 发现高质量 相对领导一成员交换增加员工顾全大局式建言行为 [14]。

因而, 本研究推断当员工拥有高质量领导一成员交换 关系, 更愿意做出建言行为。即:

H1：LMX与促进性建言行为正相关。

H2：LMX与抑制性建言行为正相关。

\section{2. 心理授权的中介作用}

\subsection{1. 心理授权}

长久以来, 关于授权的研究一直组织行为学家的研究 热点。总体而言, 对于授权的研究可分为两种视角: 宏观 视角与微观视角。前者关注组织中自上而下的授权, 也被 称为结构授权, 后者从员工心理角度出发, 关注个体心理 知觉。随着研究的深入, 部分学者逐渐认识到传统授权仅 关注上级将权力授予下属, 却忽视被授权者心理感受 [15]。 然而只有当个体感觉到 “被授权” 时，授权才有效。即授 权举措作用的发挥离不开被授权者心理感受。基于这种认 识, Thomas等人 (1990) 在前人研究基础上提出心理授权 (psychological empowerment) 这一概念。他们认为心 理授权是个体体验的心理状态或认知的综合体。是包括工 作和个体价值相匹配的心理认知 [16]。心理授权共包括四 个维度：工作意义（工作目标的价值）, 自我效能感和胜 任力（感知到完成工作相关的任务的能力），工作自主性 (能够发起行动) 以及工作影响 (个体能够影响工作结果 的程度）。Speritzer（1995）进一步验证了这 4 个维度, 并且编制了心理授权量表 [17]。我国学者李超平等 (2006) 验证了Speritzer的量表, 结果表明该量表在中国情景下 同样适用[18]。

\subsubsection{LMX与心理授权}

高质量的领导一成员交换关系能够有有效的激发员工 心理授权。具体而言, 首先, 高LMX能够使员工感知到他们 对于组织的重要性, 还能够为他们从领导处获得更多的信 息。这能够帮助员工理解其工作意义; 其次, 在高质量的 领导一成员交换关系中，领导者经常鼓励员工，表露出对 其高任务绩效的信心，从而提高员工对自我价值的感知, 并更有可能更加积极的承担起他们的工作 [19]。简而言之, 
领导的鼓励有助于员工自我效能感知的形成，增强对自身 能力的感知; 第三, 对高LMX者而言, 来自领导的信任, 可 以使得他们被允许参与决策制定, 以及被授予决定自己工 作的自主权, 这都会增强员工对自主性的感知。最后, 高 LMX员工被允许参与决策制定, 这一过程会给予员工对于工 作的控制感, 增强员工感知到他们的行为对工作结果有影 响的知觉。综合上述理论推导, 本文提出以下假设:

H3：LMX与员工心理授权正相关。

\subsection{3. 心理授权与员工建言}

从现有研究结果来看, 个体的心理授权会对工作态度 和工作行为产生重要影响 [5]。西理授权是个体体验到的 被授权的心理状态与认知综合体, 当个体感知到被授权后, 更能激发个体的内在工作动机, 从而表现出积极的工作态 度与行为。心理授权能够有效促进个体建言行为, 具体而 言: 从工作意义角度来说, 当员工认为自身工作对组织及 他人有重要意义, 有助于实现自身的目标与价值。依据社 会交换理论, 为了对组织予以回报, 他们也将表现出更多 的积极、主动的行为, 如建言行为等角色外行为。从自我 效能感角度来说, 高自我效能感能够激发员工内在工作动 机, 个体愿意为组织利益付出额外的努力, 因而员工也乐 于为组织建言献策; 从工作自主性角度来说, 高工作自主 性使得员工感知对工作的控制感，他们希望通过提出建议 等方式来影响工作结果, 并提升现有工作状况 [20]; 从工 作影响角度来说, 员工对组织的影响力使得员工有机会参 与组织的决策, 这也使得员工有机会对组织提出建设性意 见。据此，本文提出以下假设:

H4：心理授权与员工促进性建言行为正相关。

H5：心理授权与员工抑制性建言行为正相关。

\subsection{4. 心理授权的中介作用}

LMX可以通过提升个体的心理授权进而激发员工的建 言行为。当LMX较高时, 意味着领导与员工保持着高质量 的交换关系, 员工不仅能够得到领导的信任, 而且还能够 得到反馈、资源、授权的有形和无形的支持。来自领导信 任与支持使得员工感到自己工作是有意义的, 这也有助于 激发员工的自我效能感，员工表现更为自主，也能认识到 自己对工作的影响。简而言之, 高LMX会增强员工的心理 授权。当员工感知到被授权的体验时, 其内在的工作动机 被激发, 从在工作中更加积极与主动, 也会表现出更多的 建言行为。

因此, 本文提出以下假设

H6: 心理授权在LMX与员工促进性建言行为之间起中 介作用。

H7: 心理授权在LMX与员工抑制性建言行为之间起中 介作用。

据此, 本文的提出以下研究模型, 如图1所示:

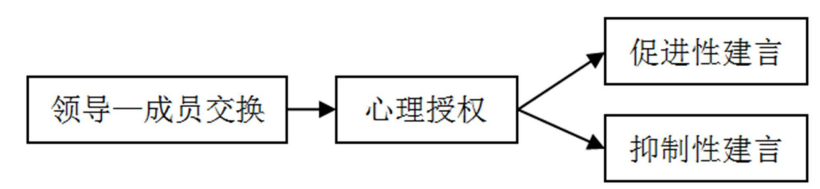

图1 理论模型。

\section{3. 研究方法}

\section{1 . 研究对象}

本研究主要共向安徽、上海等地发放问卷 300 份, 回 收250份, 在剔除信息缺失等无效问卷后, 最终获得有效 问卷 216 份。问卷有效回收率 $72 \%$ 。样本的描述性统计显示: 从性别看, 男性占 $54 \%$, 女性占 $46 \%$ 。从年龄上看, 25 岁以 下占 $13 \%, 26-35$ 岁占 $32.4 \%, 36-45$ 岁占 $36.1 \%, 46-55$ 占 $16.7 \%, 56$ 岁及以上占 $1.9 \%$, 从教育程度上看, 专科及 以下占 $6.5 \%$, 本科占 $69.4 \%$, 硕士研究生及以上占 $24.1 \%$, 从职位等级上看, 普通员工占 $21.3 \%$, 基层管理者占 $13.9 \%$, 中层管理者占 $30.6 \%$, 高层管理者占 $34.3 \%$ 。

\section{2. 测量工具}

本研究采用的变量为领导一成员交换, 心理授权及员 工建言, 其测量工具具体如下,

（1）领导一成员交换，采用王辉等2004年开发的量 表, 该量表是在Liden和Maslyn编制的12题目领导成员交 换量表基础上, 结合中国情景修订而成。共包含 16 个题项, 具有代表性的测量题项包括“我非常喜欢我主管的为人”, “我愿意为我主管做超出我职责范畴之外的工作” 等。量 表在本研究中的Cronbach' $\mathrm{s}$ a 系数为 0.87 , 表明该量表 具有良好的信度。

（2）员工心理授权，该量表是Spreitzer1997年编制, 得到学者们的广泛认同。我国学者李超平等2006年的研究 表明该量表在中国情境下具有较好的信度和效度。具有代 表性的题项包括: “我所做的工作对我来说非常有意义”,

“我对自己完成工作的能力非常有信心”。量表在本研究 中的Cronbach' $\mathrm{s}$ a 系数为 0.82 。

(3) 建言行为, 采用Liang等开发的二维度量表, 共 包括 11 个题项，典型题项如 “. 当单位内的工作出现问题 时, 我敢于指出, 不怕得罪人”, “我就改善单位工作程 序积极地提出了建议”。量表在本研究中的Cronbach' $\mathrm{s}$ a 系数为 0.81 。

本文所有量表均采用李克特5点计分法, 1 代表 “完全 不同意”，5代表 “完全同意”。

\section{4. 数据统计与假设检验}

\section{1. 同源方差分析}

由于本研究所设计的问卷所有题项都是由同一被试 者完成, 为了消除可能存在的同源偏差 (Common Method Variance), 我们采用哈曼单因子检测方法, 将所有题项 一起做因子分析。统计结果表明, 未旋转首因子载荷量为 $35.5 \%$, 并未占到大多数 (总量为 $76.5 \%$ ), 同源方差问题 并不严重。

\section{2. 效度分析}

本研究还对变量的聚合效度与区分效度进行了检验。 验证性因子分析结果表明, 各量表因子载荷均在 0.5 以上, 因此, 量表具有较好的聚合效度。本研究采用LISREL8.7 
进行 CFA 检验, 选取主要指标的测量结果如下: RMSEA=0. 037; 由此可以认为本研究采用量表具有较好的 $\mathrm{x}^{2} / \mathrm{df}=1.14<2, \mathrm{GFI}=0.86, \mathrm{CFI}=0.99, \mathrm{NFI}=0.93$, 效度。

\section{3. 变量之间相关分析}

本研究中各变量描述性统计及变量间相关系数见表 1 。结果表明LMX与抑制性建言正相关 $(r=0.438, P<0.01)$; 与 促进性建言正相关（ $r=0.419, P<0.01 ） ;$ 与心理授权正相关（ $r=0.441, P<0.01 ）$ ；心理授权与与抑制性建言正相关 $(r=0.556, P<0.01) ;$ 与促进性建言正相关（ $r=0.616, P<0.01 ） 。$

表1 各变量描述性统计、相关系数。

\begin{tabular}{|c|c|c|c|c|c|c|c|c|c|c|c|}
\hline 变量 & 均值 & 标准差 & 1 & 2 & 3 & 4 & 5 & 6 & 7 & 8 & 9 \\
\hline 1. 性别 & 0.600 & 0.492 & 1.000 & & & & & & & & \\
\hline 2. 年龄 & 2. 620 & 0.974 & 0.072 & 1. 000 & & & & & & & \\
\hline 3. 学历 & 2. 180 & 0.526 & 0.020 & $0.277 * *$ & 1. 000 & & & & & & \\
\hline 4. 工作年限 & 3.780 & 1. 138 & -0.043 & $0.825 * *$ & 0.175 & 1.000 & & & & & \\
\hline 5. 组织类型 & 3. 160 & 1. 692 & 0.098 & 0.020 & 0.021 & 0.067 & 1. 000 & & & & \\
\hline 6. LMX & 3. 623 & 0.582 & -0.018 & -0.098 & 0.091 & -0.074 & -0.039 & $(0.87)$ & & & \\
\hline 7. PE & 3. 793 & 0.602 & 0.082 & $0.209 *$ & 0.128 & $0.249 * *$ & 0.067 & $0.441 * *$ & $(0.82)$ & & \\
\hline 8. 抑制性建言 & 3. 397 & 0.640 & -0.038 & 0.049 & 0.138 & 0.094 & -0.160 & $0.438 * *$ & $0.556 * *$ & $(0.81)$ & \\
\hline 9. 促进性建言 & 3. 767 & 0.574 & 0.072 & 0.091 & 0.026 & $.206 *$ & 0.030 & $0.419 * *$ & $0.616 * *$ & $0.686 * *$ & $(0.81)$ \\
\hline
\end{tabular}

注: $* *$ 表示 $\mathrm{P}<0.01 ; *$ 表示 $\mathrm{P}<0.05$, 对角线上括号内为Cronbach' $\mathrm{s}$ a 系数

\section{4. 假设检验}

本研究使用层次回归方法检验本文的研究假设, 如下 表所示。具体而言，模型 1 以心理授权为因变量，对相关 控制变量进行回归, 模型 2 在模型 1 基础上, 引入领导一成 员交换, 结果表明领导一成员交换对心理授权有显著正向 影响（r=0.464， p <0.001）。假设H3得到支持。

模型 3 以促进性建言为因变量对控制变量进行回归, 模型 4 中, 引入领导一成员交换为自变量, 检验结果表明 领导一成员交换对促进性建言有显著的正向预测作用 $(\mathrm{r}=0.435, \mathrm{p}<0.001)$ ，假设 $\mathrm{H} 1$ 得到验证。模型 5 在模型 3 基础上引入心理授权, 检验结果表明心理授权对促进性建 言显著的正向影响（r=0.601，p $<0.001 ）$, 假设 $H 4$ 得到验 证;
模型7以抑制性建言为因变量对控制变量进行回归, 模型8引入领导一成员交换为自变量, 结果表明领导一成 员交换对员工抑制性建言有显著正向影响 $(r=0.429$, $\mathrm{p}<0.001$ ），假设 $\mathrm{H} 2$ 得到支持，模型 9 在模型 7 基础上引入心 理授权，结果显示，心理授权对员工抑制性建言显著正向 影响（ $\mathrm{r}=0.576, \mathrm{p}<0.001 ）$ ，假设 $\mathrm{H} 5$ 得到验证;

模型6中, 在加入心理授权这一中介变量之后, LMX 对促进性建言的预测作用仍然显著, 表明心理授权在领导 一成员交换与促进性建言之间起到部分中介作用。假设H6 得到部分支持。同理，模型10也表明心理授权在领导一成 员交换与抑制性建言之间起到部分中介作用，假设H7得到 部分支持。

表2 多层回归分析结果（N=216）

\begin{tabular}{|c|c|c|c|c|c|c|c|c|c|c|}
\hline \multirow{2}{*}{ 变量 } & \multicolumn{2}{|c|}{ 心理授权 } & \multicolumn{4}{|c|}{ 促进性建言 } & \multicolumn{4}{|c|}{ 抑制性建言 } \\
\hline & 模型1 & 模型2 & 模型3 & 模型4 & 模型5 & 模型6 & 模型7 & 模型8 & 模型9 & 模型10 \\
\hline 性别 & 0.093 & 0.093 & 0.112 & 0.113 & 0.057 & 0.066 & -0.020 & -0.001 & -0.055 & -0.046 \\
\hline 年龄 & -0.055 & 0.025 & -0.298 & -0.223 & -0.265 & -0.235 & -0.172 & -0.098 & -0.140 & -0.110 \\
\hline 学历 & 0.092 & 0.031 & 0.027 & -0.030 & -0.028 & -0.046 & 0.150 & 0.094 & 0.098 & 0.080 \\
\hline 工作年限 & 0.280 & 0.258 & $0.452 *$ & $0.431 * *$ & $0.284 *$ & $0.302 *$ & 0.222 & 0.201 & 0.060 & 0.079 \\
\hline 组织类型 & 0.038 & 0.057 & $-0.006 *$ & 0.013 & -0.028 & -0.016 & -0.175 & -0.157 & -0.197 & $-0.184 *$ \\
\hline LMX & & $0.464 * * *$ & & $0.435 * * *$ & & $0.202 *$ & & $0.429 * * *$ & & $0.209 *$ \\
\hline PE & & & & & $0.601 * * *$ & $0.502 * * *$ & & & $0.576 * * *$ & $0.473 * * *$ \\
\hline F值 & 1. 781 & 6.855 & 1. 647 & 5.876 & & 11. 138 & 1. 332 & 5. 317 & 9.723 & 9. 487 \\
\hline $\mathrm{R}^{2}$ 值 & 0.080 & 0.289 & 0.075 & 0.259 & 0.407 & 0.438 & 0.061 & 0.240 & 0.366 & 0.399 \\
\hline$\Delta \mathrm{R}^{2}$ & & 0.209 & & 0.184 & 0.332 & 0.363 & & 0.179 & 0.305 & 0.338 \\
\hline
\end{tabular}

注: ***表示 $\mathrm{P}<0.001 ; * *$ 表示 $\mathrm{P}<0.01 ; *$ 表示 $\mathrm{P}<0.05$

\section{5. 讨论与结论}

\section{1. 研究结论与贡献}

本研究以上海、安徽等地 216 名员工为研究样本，探 讨了LMX对员工促进性建言和抑制性建言的影响及其作用
机制。具体而言, 首先, 本文的研究结果表明LMX对员工 促进性建言和抑制性建言均有显著正向影响。本文从关系 视角出发验证了LMX对员工建言行为的重要影响, 突破了 以往从领导风格、员工特质视角研究员工建言行为的局限 性, 证实了领导与员工的互动对于员工建言行为的影响。 高质量的LMX关系不仅使得员工拥有更多与领导交流、沟 
通的机会，还能得到领导的信任与支持，从而激发员工促 进性与抑制性的建言; 其次, LMX对心理授权具有显著的 正向影响, 高质量的LMX能够激发员工的心理授权; 最后, 本文证实心理授权在LMX与员工建言之间起部分中介作用。 高质量的领导一成员交换关系会使得员工感知到被授权， 进而激发员工的建言行为。反之, 当领导一成员交换关系 较差, 员工不能感知到被授权, 会抑制员工建言行为的发 生。心理授权中介机制的揭示了中国情景下建言行为的发 生机制, 也丰富了心理授权的相关研究。

针对企业管理实践, 本研究的建议有:

一方面, 企业需要构建良好的上下级关系。本文的研 究结果表明LMX是促进下属建言的重要因素, 尤其在注重 关系的中国社会, 与领导关系质量较高的圈内员工他们更 愿意为企业建言献策。因而, 通过构建良好的上下级关系, 增强上下级之间的沟通、信任，打消员工建言时的顾虑。 在此基础上, 企业还应该为员工营造良好的沟通机制, 让 员工能言、敢言、善言。另一方面, 领导者应提高员工心 理授权的感知。领导者应当增强员工的心理授权, 换言之, 要让员工认识到自己的工作的价值, 以及员工在自己工作 中的自主性, 还要让员工认识到他们对工作结果的影响力 等, 从而激发员工的为企业建言的动机。

\section{2. 研究不足与展望}

首先, 由于横截面的研究数据的局限性使得本研究只 能得到变量间的相关关系, 未来应当通过纵向的研究进一 步验证。其次, 样本的局限性。由于样本获取的限制, 使 得本研究的样本集中在安徽、上海等地区, 因而本文的研 究结论在其他地区能否得到支持有待讨论。最后, 本研究 并未探讨LMX各维度对心理授权的影响, 也未探讨心理授 权各维度在LMX与建言行为之间的中介作用。未来研究可 以不断深入、细化。

未来研究, 首先可以选择其他情境对本研究进行验证, 其次还可以继续研究中国文化要素对建言行为的影响, 如 中国文化中，“不患寡而患不均” 等中庸思想的影响下， 组织公正感等是否会对员工建言产生影响。这些中国文化 要素都值得进一步研究, 从而为激发员工建言提供有益指 导。

\section{参考文献}

[1] 李锐, 凌文轻, 柳士顺. 上司不当督导对下属建言行为的影 响及其作用机制 [J]. 心理学报, 2009, 12期: 1189-1202。

[2] Crant J M. Proactive Behavior in Organizations [J]. Journal of Management, 2000, 26 (3) :435-462.

[3] Milliken F J, Morrison E W, Hewlin P F. An Exploratory Study of Employee Silence: Issues that Employees Don't Communicate Upward and Why $[\mathrm{J}]$. Journal of Management Studies, 2003, 40 (6) :1453-1476.

[4] 向常春, 龙立荣. 参与型领导与员工建言: 积极印象管理动 机的中介作用 $[J]$. 管理评论, 2013, 25。
[5] 凌俐, 陆昌勤. 心理授权研究的现状 [J]. 心理科学进 展, 2007, 04期 (15:4) :652-658。

[6] Graen G, Dansereau F, Minami T. Dysfunctional leadership styles [J]. Organizational Behavior \& Human Performance, 1972, 7 (2) : 216-236.

[7] Green S G, Anderson S E, Shivers S L. Demographic and Organizational Influences on Leader-Member Exchange and Related Work Attitudes [J]. Organizational Behavior \& Human Decision Processes, 1996, 66 (2): $203-214$.

[8] Dyne L V, Lepine J A. HELPING AND VOICE EXTRA-ROLE BEHAVIORS: EVIDENCE OF CONSTRUCT AND PREDICTIVE VALIDITY $[J]$. Academy of Management Journal, 1998, 41 (1) : $108-119$.

[9] Dyne L V, Ang S, Botero I C. Conceptualizing Employee Silence and Employee Voice as Multidimensional Constructs [J]. Journal of Management Studies, 2003, 40 (6) : 1359-1392.

[10] Liang J, Farh J L. Promotive and prohibitive voice behavior in organizations: A two-wave longitudinal examination $[\mathrm{C}] / / 2008$.

[11] Gerstner C R, Day D V. Meta-Analytic review of leader-member exchange theory: Correlates and construct issues. [J]. Journal of Applied Psychology, 1997, 82 (6) : 827-844.

[12] 汪林, 储小平, 倪婧. 领导-部属交换、内部人身份认知与组 织公民行为一基于本土家族企业视角的经验研究 $[\mathrm{C}] / /$ 创 业与家族企业国际研讨会. 2008。

[13] 钱士茹, 丁明明, 孔苓, 等. 领导一一部属交换、内部人身份 认知与员工建言一一以组织身份吸引力为调节的实证研究 $[J]$. 科学决策, 2015(4):1-20。

[14] 再霞, 杨倩. 相对领导-成员交换对员工建言行为的影响 [J]. 商业研究, 2015, 05期: 130-136。

[15] Conger J A, Kanungo R N. The Empowerment Process: Integrating Theory and Practice $[\mathrm{J}]$. Academy of Management Review, 1988, 13 (3) : 471-482.

[16] Thomas K W, Velthouse B A. Cognitive Elements of Empowerment: An "Interpretive" Model of Intrinsic Task Motivation[J]. Academy of Management Review, 1990, 15 (4) : 666-681.

[17] Spreitzer G M. Psychological Empowerment in the Workplace: Dimensions, Measurement, and Validation[J]. Academy of Management Journal, 1995, 38 (5) : 1442-1465.

[18］Li CP (李超平), Li XX (李晓轩), Shi K (时勘), 等. 授权 的测量及其与员工工作态度的关系; Psychological Empowerment: Measurement and its Effect on Employee Work Attitude in China [J]. 心理学报, 2006, 38: 99-106. 
[19] Gist M E, Mitchell T R. Self-Efficacy: A Theoretical Analysis of Its Determinants and Malleability [J]. Academy of Management Review, 1992, 17 (2) :183-211.
[20] Frazier M L, Fainshmidt S. Voice Climate, Work Outcomes, and the Mediating Role of Psychological Empowerment A Multilevel Examination [J]. Group \& Organization Management, 2012, 37 (6) : 691-715. 